\title{
Phytoprotection
}

\section{Utilisation d'une souche indigène de Trichoderma harzianum contre cinq agents pathogènes chez le concombre et la tomate de serre au Québec \\ Use of an indigenous strain of Trichoderma harzianum against five plant pathogens on greenhouse cucumber and tomato in Québec}

\author{
J. Caron, L. Laverdière, P.O. Thibodeau et R.R. Bélanger
}

Volume 83, numéro 2, 2002

URI : https://id.erudit.org/iderudit/706230ar

DOI : https://doi.org/10.7202/706230ar

Aller au sommaire du numéro

Éditeur(s)

Société de protection des plantes du Québec (SPPQ)l

ISSN

0031-9511 (imprimé)

1710-1603 (numérique)

Découvrir la revue

Citer cet article

Caron, J., Laverdière, L., Thibodeau, P. \& Bélanger, R. (2002). Utilisation d'une souche indigène de Trichoderma harzianum contre cinq agents pathogènes chez le concombre et la tomate de serre au Québec. Phytoprotection, 83(2), 73-87. https://doi.org/10.7202/706230ar
Résumé de l'article

Le potentiel antagoniste du biofongicide à base de Trichoderma harzianum MAUL-20, isolé au Québec, a été testé contre cinq agents telluriques phytopathogènes(Fusarium oxysporum f. sp. radicis-lycopersici (FORL), Pythium ultimum, Rhizoctonia solani, Sclerotinia sclerotiorum et Verticillium dahliae) du concombre et de la tomate de serre. Le biofongicide a démontré une efficacité contre $P$. ultimum et $R$. solani chez le concombre et la tomate et contre FORL chez la tomate. De plus, $T$. harzianum MAUL-20 a eu un effet stimulant sur le développement des plants de concombre lorsque cultivés, sans agents pathogènes, dans un substrat organique alimenté du biofongicide. L'efficacité de $T$. harzianum MAUL-20 a été comparée à celle du biofongicide américain Rootshield ${ }^{\mathrm{TM}}$ (Trichoderma harzianum KRL-AG2) et le premier a démontré une activité antagoniste égale ou supérieure à celle de Rootshield ${ }^{\mathrm{TM}}$. 


\title{
Utilisation d'une souche indigène de Trichoderma harzianum contre cinq agents pathogènes chez le concombre et la tomate de serre au Québec
}

\author{
Johanne Caron ${ }^{1}$, Lucie Laverdière ${ }^{1}$, Pierre O. Thibodeau ${ }^{2}$ \\ et Richard R. Bélanger ${ }^{3}$
}

Reçu 2002-04-22; accepté 2002-07-23

PHYTOPROTECTION $83: 73-87$

Le potentiel antagoniste du biofongicide à base de Trichoderma harzianum MAUL-20, isolé au Québec, a été testé contre cinq agents telluriques phytopathogènes (Fusarium oxysporum f. sp. radicis-lycopersici (FORL), Pythium ultimum, Rhizoctonia solani, Sclerotinia sclerotiorum et Verticillium dahliae) du concombre et de la tomate de serre. Le biofongicide a démontré une efficacité contre $P$. ultimum et $R$. solani chez le concombre et la tomate et contre FORL chez la tomate. De plus, T. harzianum MAUL-20 a eu un effet stimulant sur le développement des plants de concombre lorsque cultivés, sans agents pathogènes, dans un substrat organique alimenté du biofongicide. L'efficacité de T. harzianum MAUL-20 a été comparée à celle du biofongicide américain Rootshield ${ }^{\mathrm{TM}}$ (Trichoderma harzianum KRL-AG2) et le premier a démontré une activité antagoniste égale ou supérieure à celle de Rootshield ${ }^{\mathrm{TM}}$.

\section{[Use of an indigenous strain of Trichoderma harzianum against five} plant pathogens on greenhouse cucumber and tomato in Québec]

Trichoderma harzianum MAUL-20, a strain isolated from a soil in the province of Québec, was evaluated for its antagonistic potential against five plant pathogens (Fusarium oxysporum f. sp. radicis-lycopersici (FORL), Pythium ultimum, Rhizoctonia solani, Sclerotinia sclerotiorum, and Verticillium dahliae) on greenhouse cucumber and tomato. It reduced disease incidence significantly against $P$. ultimum and $R$. solani on both cucumber and tomato and against FORL on tomato. In addition, $T$. harzianum MAUL20 stimulated plant growth of cucumber plants when amended to a substrate without plant pathogens. Its efficacy was compared to that of Rootshield ${ }^{\mathrm{TM}}$, a biofungicide based on Trichoderma harzianum KRL-AG2 registered in the USA. Its biocontrol potential was equivalent or superior to Rootshield ${ }^{\mathrm{TM}}$.

1. Horti-Protection inc., 11 rue des Peupliers, Sainte-Hélène de Breakeyville (Québec), Canada G0S 1E1; courriel : hortipro@mediom.qc.ca

2. Institut de Recherche et de Développement en Agroenvironnement, Complexe scientifique, 2700, rue Einstein, Sainte-Foy (Québec), Canada G1P 3W8

3. Centre de Recherche en Horticulture, Département de Phytologie, Université Laval, Québec (Québec), Canada G1K 7P4 


\section{INTRODUCTION}

La microflore et la microfaune utiles et nuisibles du sol sont très importantes et diversifiées à différents niveaux de profondeur dans le sol. La plupart des micro-organismes sont utiles, tandis que d'autres constituent de véritables ennemis et entravent le développement de la plante et de son système racinaire. Certains agents telluriques phytopathogènes du sol sont responsables d'importantes pertes de récoltes en causant diverses maladies dont : 1) pourriture des semences (Fusarium spp., Pythium spp., etc.), 2) fonte des semis (Pythium spp., Sclerotinia spp., etc.), 3) pourriture racinaire (Fusarium spp., Phytophthora spp., Rhizoctonia spp., etc.) et 4) flétrissement des plantes (Fusarium spp., Verticillium spp., etc.) (Agrios 1988; Anonyme 1992). Les pertes moyennes estimées par l'action de ces micro-organismes nuisibles sur les racines représentent environ $15 \%$ de la production agricole (Agrios 1988) et, dans le cas de complexes d'agents pathogènes, des pertes allant jusqu'à $60 \%$ ont été rapportées (Sippell et al. 1985). Pour réduire l'importance de ces ennemis, les producteurs ont souvent recours à tout un arsenal de pesticides (Jarvis 1993; Lumsden et Lewis 1989; Ole Becker et Schwinn 1993) et de fumigants (Chet et Baker 1981; Larkin et Fravel 1998) avec des résultats souvent mitigés (Besnard et Davet 1993; De Waard et al. 1993; Jarvis 1993) et des effets secondaires sur l'environnement et les organismes utiles parce que ces produits sont non sélectifs.

Les maladies racinaires sont complexes et difficiles à combattre parce que souvent initiées par les blessures causées par les insectes et nématodes nuisibles qui facilitent l'entrée et la progression des champignons. Cette situation conduit à une utilisation abusive de pesticides chimiques de synthèse, qui polluent inutilement, créent des conditions favorables au développement de la résistance des ennemis (insectes, nématodes et champignons) à ces produits et détruisent les organismes telluriques utiles (Harman 1992).
Plusieurs micro-organismes telluriques peuvent, par contre, avoir un effet bénéfique dans le contrôle des champignons pathogènes des racines et ainsi minimiser l'effet délétère des pesticides sur la rhizosphère (Adams 1990). La découverte de tels agents de lutte biologique et la démonstration de leur capacité à réduire l'incidence et la gravité des maladies ont tracé la voie à plusieurs recherches prometteuses (Alabouvette et al. 1993; Bélanger et Labbé 1994; Boland 1990; Lewis et Papavizas 1987). Dans ce contexte, une attention particulière a été portée aux champignons antagonistes, principalement à cause de leur potentiel à diminuer la densité de l'inoculum des champignons pathogènes (Dennis et Webster 1971b; Elad et al. 1982; Larkin et Fravel 1998; Lockwood 1988). Parmi les champignons antagonistes qui ont démontré un bon potentiel de lutte, Trichoderma spp. est sans contredit le plus rapporté dans la littérature (Elad et al. 1982; Harman 2000) ayant démontré des effets contre : Pythium (Bolton 1980; Chet et al. 1981; Clavet et al. 1993); Phytophthora cinnamomi Rands (Kelley 1976), Rhizoctonia solani Kühn (Elad et al. 1980; Lewis et Papavizas 1987; Windham et al. 1986); Sclerotium rolfsii Saccardo (Backman et RodriguezKabana 1975), etc. Ces champignons pathogènes causent d'importants dégâts dans les cultures fruitières, légumières, serricoles et ornementales en s'attaquant particulièrement aux racines et au collet de ces cultures.

Trichoderma spp. est naturellement abondant dans le sol et la matière organique tels le bois mort ou en décomposition, les débris végétaux et la paille (Papavizas 1985; Sippell et al. 1985; Widden et Scattolin 1988). Les espèces de ce genre possèdent également des aptitudes à dégrader de nombreux substrats organiques du sol pour se nourrir et se développer ce qui suggère qu'elles peuvent survivre dans plusieurs niches écologiques (Papavizas 1985). Certains substrats vendus commercialement contiennent déjà de tels ingrédients organiques ce qui faciliterait le développement du champignon au sein de ces substrats. Alors que les produits de lutte biologique contre les maladies 
tardent à s'implanter commercialement (Harman 2000), ceux développés contre les insectes ravageurs retrouvés en serre sont maintenant généralisés et performants (ex: Amblyseius cucumeris Oudemans [Acari : Phytoseiidae], Encarsia formosa Gahan [Hymenoptera: Aphelinidae], Phytoseiulus persimilis Athias-Henriot [Acari : Phytoseiidae], etc.) (Brodeur et al. 1997; Cook 1993; Gill et Sanderson 1998).

Lors de travaux précédents, Caron (1993) a évalué le potentiel antagoniste de 142 souches indigènes de Trichoderma isolées à partir de sols provenant de différentes régions agricoles du Québec. De toutes ces souches, Trichoderma harzianum Rifai MAUL-20 fut la souche retenue en raison de son développement adapté aux conditions climatiques observées dans les serres au Québec. Son fort potentiel antagoniste contre Botrytis cinerea Pers.:Fr., un champignon pathogène des organes aériens de la tomate, a été démontré sur des plants cultivés en serre au Québec (Caron, résultats non publiés). Les résultats prometteurs obtenus incitaient à explorer une autre facette de son potentiel soit la lutte contre les agents pathogènes présents dans les substrats. L'objectif général de cette étude était d'évaluer le potentiel antagoniste de $T$. harzianum MAUL-20 contre plusieurs champignons pathogènes des substrats en cultures de concombre et de tomate.

\section{MATÉRIEL ET MÉTHODES}

\section{Champignons pathogènes}

Les champignons pathogènes utilisés dans cette recherche incluaient des souches de Fusarium oxysporum Schlecht. ex Fries f. sp. radicis-lycopersici W.R. Jarvis \& Shoemaker (FORL), Rhizoctonia solani (AG-3), Sclerotinia sclerotiorum (Lib.) de Bary, Verticillium dahliae Kleb. et Pythium ultimum Trow. Les quatre premiers champignons ont été maintenus sur un milieu gélosé à base de pomme de terre (PDA, Difco, Détroit, $\mathrm{MI}$ ) tandis que $P$. ultimum a été cultivé sur le milieu $P_{5}$ ARP (17 g Cornmeal agar (Difco), 0,005 g de pimaricine (Sigma, St-Louis, MO), 0,25 g d'ampi- cilline (Sigma), 0,010 $\mathrm{g}$ de rifampicine (Sigma), 0,100 g de pentachloronitrobenzène (PCNB) (Sigma) et $800 \mathrm{~mL}$ d'eau distillée par litre) (Jeffers et Martin 1986). Les souches de FORL, $R$. solani et $V$. dahliae ont été fournies par M. Mario Tésolin du laboratoire de diagnostic en phytoprotection du ministère de I'Agricultures, des Pêcheries et de l'Alimentaion du Québec (MAPAQ); $P$. ultimum a été obtenu de HortiProtection inc. et $S$. sclerotiorum de Mme Sylvie Rioux du Centre de recherche sur les grains inc. (CEROM).

\section{Champignons antagonistes}

L'agent de lutte biologique Trichoderma harzianum MAUL-20 a été isolé du sol provenant de la région agricole de I'Assomption au Québec. Cette souche fait partie d'une banque québécoise de 142 souches de Trichoderma. Trichoderma harzianum MAUL-20 a été maintenu sur un milieu PDA par des repiquages réguliers.

Le biofongicide Rootshield ${ }^{\mathrm{TM}}$ (BioWorks Inc., Geneva, NY) a été fourni par le Dr C. Hayes de la compagnie BioWorks Inc., pour fins de recherche. Ce biofongicide à base de Trichoderma harzianum KRL-AG2 (souche T-22) est homologué et commercialisé aux ÉtatsUnis pour les cultures en serre, l'industrie des gazons et les cultures légumières de champ. II a, entre autres, une activité antagoniste rapportée contre Pythium, Rhizoctonia et Fusarium (Harman 2000).

\section{Production des inocula}

Les inocula de Trichoderma harzianum MAUL-20 et des cinq champignons pathogènes ont tous été produits en masse dans des plateaux en aluminium pré-stérilisés $(52.71 \mathrm{~cm} \times 32.39 \mathrm{~cm} \times$ $3.81 \mathrm{~cm}$ ) (Alcan Aluminium Itée, Montréal, Canada), munis d'un couvercle, et contenant $675 \mathrm{~mL}$ de milieu spécifique à chacun des organismes étudiés.

L'inoculum de $T$. harzianum MAUL20 a été produit sur un milieu V-8 contenant par litre de milieu, $200 \mathrm{~mL}$ de jus V-8 non modifié (Les Soupes Campbell Itée, Ontario, Canada), $3 \mathrm{~g}$ de $\mathrm{CaCO}_{3}$ (Les Produits chimiques Omega inc., Québec), $8 \mathrm{~g} \mathrm{~d}$ 'agar (Difco) et $800 \mathrm{~mL}$ d'eau distillée. Le milieu a été auto- 
clavé $30 \mathrm{~min}$, refroidi à $42^{\circ} \mathrm{C}$, puis ensemencé avec une suspension sporale de $T$. harzianum MAUL-20 $\left(2 \times 10^{11} \mathrm{cfu}\right.$ $\left.\mathrm{mL}^{-1}\right)$. Ce milieu a été versé dans les plateaux d'aluminium et incubé $6 \mathrm{j}$ à 22 $\pm 2^{\circ} \mathrm{C}$, à la noirceur. Les spores ont été récoltées en grattant la surface de la culture préalablement inondée d'eau distillée stérile et la suspension a été homogénéisée dans un mélangeur domestique (Osterizer, Sunbeam, Canada) pendant $1 \mathrm{~min}$ et ensuite mélangée à de l'argile brune (Les revêtements en poudre Permalux inc., Montréal, Québec) ( $4: 1 \mathrm{w}: \mathrm{v})$. La formulation a été lyophilisée dans des plateaux en acier inoxydable (Virtis, Gardiner, NY) pendant $48 \mathrm{~h}$ à $-60^{\circ} \mathrm{C}$, moulue, ensachée et mise sous vide. Le produit final titrait en moyenne $1 \times 10^{8}$ spores de $T$. harzianum $\mathrm{g}^{-1}$ de poudre inoculante.

Le biofongicide Rootshield est disponible commercialement aux ÉtatsUnis sous forme de poudre mouillable. Il a été appliqué au substrat à la concentration recommandée par le fabriquant $\left(1,15 \% ; 1,0 \times 10^{7} \mathrm{cfu} \mathrm{g}^{-1}\right)$.

La production du $V$. dahliae et de FORL a été réalisée sur un milieu PDA et incubée $7 \mathrm{j}$ à $22 \pm 2^{\circ} \mathrm{C}$. Les spores furent récoltées, mélangées à l'argile brune et traitées tel que décrit pour $T$. harzianum MAUL-20. La concentration en spores de la formulation finale des deux champignons se situait autour de $1 \times 10^{7}$ spores $\mathrm{g}^{-1}$.

La production d'inoculum de $R$. solani et $S$. sclerotiorum a été réalisée sur milieu PDBroth $^{++}: 24 \mathrm{~g}$ de PDBroth (Difco) et $8 \mathrm{~g}$ d'agar (Difco) par litre et incubée $5 \mathrm{j}$ dans le noir, à $22 \pm 2^{\circ} \mathrm{C}$. La culture entière fut homogénéisée à l'aide d'un mélangeur domestique pendant $1 \mathrm{~min}$ et la concentration finale de propagules (fragments d'hyphes et de cellules monilioïdes dans le cas de $R$. solani et fragments d'hyphes et de sclérotes pour $S$. sclerotiorum) a été évaluée à l'aide d'un hémacytomètre (C.A. Hausser and son, Philadelphie, PA) et ajustée à $1 \times 10^{3}$ propagules $\mathrm{mL}^{-1}$, par dilution dans de l'eau distillée stérile. L'inoculum a été utilisé immédiatement.
L'inoculum de Pythium a été produit de la même façon que celui de $T$. harzianum MAUL-20, sauf que la suspension de P. ultimum $(30 \mathrm{~mL})$ fut étalée à la surface du milieu gélosé pré-refroidi plutôt qu'intégré au milieu tiédi. $L^{\prime}$ incubation a duré $7 \mathrm{j}$ à $22 \pm 2^{\circ} \mathrm{C}$. La culture entière a été broyée et homogénéisée au mélangeur domestique durant $1 \mathrm{~min}$ et la concentration finale de propagules (sporanges et fragments d'hyphes) ajustée avec l'hémacytomètre à $1 \times 10^{3}$ propagules $\mathrm{mL}^{-1}$, par dilution avec de l'eau distillée stérile. L'inoculum a été utilisé immédiatement.

\section{Essais en serre}

Tous les essais en serre ont été réalisés sous les conditions suivantes: 1) Cultures de plants de concombre (Cucumis sativus L. Cv. Revenue) et de tomate ( Lycopersicon esculentum Mill. cv. Beefmaster) en caissettes de styromousse $(16,5 \mathrm{~cm} \times 29,2 \mathrm{~cm} \times 7,6 \mathrm{~cm})$ contenant $2 \mathrm{~L}(400 \mathrm{~g})$ de substrat PRO-MIX "PGX" (Premier Horticulture Itée, Rivière-duLoup, Québec); 2) Ensemencement de 12 graines par caissette; 3) Durée de culture en serre de $30 \mathrm{j}$ à $22 \pm 2^{\circ} \mathrm{C}$ (jour) et $18 \pm 2^{\circ} \mathrm{C}$ (nuit) avec une photopériode de 16:8 L:N, une irrigation journalière ( $2 \mathrm{~min}$ ( $80 \mathrm{~mL}$ d'eau), deux fois par j) et une fertilisation hebdomadaire N-P-K (20-20-20); 4) Le pourcentage de germination, la hauteur et le poids frais de la partie aérienne des plantes ont été déterminés après $30 \mathrm{j}$ de croissance en serre.

\section{Effet de la concentration en spores de Trichoderma harzianum MAUL-} 20 sur le concombre et la tomate Des concentrations de $0,10^{4}, 10^{5}, 10^{6}$ et $10^{7}$ spores de $T$. harzianum MAUL-20 $\mathrm{g}^{-1}$ de sol ont été introduites en mélange au substrat PRO-MIX "PGX" afin de déterminer la quantité optimale requise pour assurer la protection du système racinaire des plants de concombre et de tomate et pour vérifier la possibilité d'effets négatifs sur la croissance des plantes utilisées. Le témoin était composé des plantes sensibles semées directement dans le substrat. Chaque traitement a été répété trois fois. 
Activité antagoniste de Trichoderma harzianum MAUL-20 contre cinq champignons telluriques phytopathogènes

L'action des différents champignons pathogènes et de l'agent de lutte biologique $T$. harzianum MAUL-20 a été évaluée en serre expérimentale sur le concombre et la tomate. L'expérience comportait 12 traitements pour chaque espèce : les agents pathogènes $R$. solani (Rs), V. dahliae (Vd), S. sclerotiorum (Ss), FORL et $P$. ultimum (Pu) inoculés avec $(+)$ ou sans $(-) T$. harzianum et deux témoins composés de plantes sensibles semées directement dans le substrat en absence d'agents pathogènes avec (Tem+) ou sans (Tem) $T$. harzianum MAUL-20. Chaque traitement a été répété trois fois.

Dans les traitements concernés, $T$. harzianum MAUL-20 a d'abord été incorporé au substrat PRO-MIX "PGX" et laissé au repos pendant deux semaines afin de permettre sa multiplication. La quantité d'inoculum de $T$. harzianum MAUL-20 a été calculée de façon à obtenir $1 \times 10^{6}$ propagules $\mathrm{g}^{-1}$ de substrat. Seule une irrigation suffisante a été maintenue ( $2 \mathrm{~min}$, deux fois par j). Après 2 semaines d'incubation, chaque champignon pathogène a été ajouté en mélange au substrat au taux de $1 \times 10^{3}$ propagules $\mathrm{g}^{-1}$ de substrat et les plantes ont été semées. Les conditions de croissance en serre ont été décrites cidessus.

Un index visuel arbitraire de la gravité de la maladie (IGM) a également permis d'évaluer le flétrissement, la pourriture racinaire et les nécroses sur chaque plante. Les plantes ont été notées sur une échelle de 1 à 10 selon leur apparence (flétrissement, jaunissement des tiges et des feuilles, pourriture racinaire, fonte des semis, etc.) où 1 représente un plant sain et 10 un plant mort.
Comparaison de l'activité biologique de Trichoderma harzianum MAUL-20 et du biofongicide Rootshield ${ }^{\text {TM }}$

La performance de T. harzianum MAUL20 a été comparée à la souche commerciale Rootshield ${ }^{\mathrm{TM}}$ homologuée aux États-Unis, contre les cinq champignons pathogènes étudiés. Les agents pathogènes $R$. solani (Rs), $V$. dahliae $(\mathrm{Vd}), S$. sclerotiorum (Ss), FORL et $P$. ultimum $(\mathrm{Pu})$ ont été inoculés soit avec $T$. harzianum MAUL-20 (20) ou Rootshield (Ro) et trois témoins composés de plantes sensibles semées directement dans le substrat : (i) en absence de Trichoderma (Tem), (ii) en présence de $T$. harzianum MAUL-20 (20), et (iii) en présence de Rootshield (Ro). Chaque traitement comportait trois répétitions.

Les deux biofongicides ont d'abord été mélangés au substrat PRO-MIX "PGX" et laissés au repos pendant 2 semaines afin de permettre leur multiplication. La quantité d'inoculum de $T$. harzianum MAUL-20 a été calculée de façon à obtenir $1 \times 10^{6}$ propagules $\mathrm{g}^{-1} \mathrm{de}$ substrat tandis que Rootshield ${ }^{\mathrm{TM}}$ a été appliqué à une concentration de $1 \times 10^{7}$ propagules $\mathrm{g}^{-1}$ de substrat, tel que recommandé sur l'étiquette. Seul un arrosage adéquat a été maintenu (2 min, deux fois par j). Deux semaines plus tard, chaque agent pathogène a été introduit en mélange au substrat et les plantes ont été semées. Pour tous les agents pathogènes, la concentration sporale inoculée au substrat a été ajustée à $1 \times 10^{3}$ propagules $\mathrm{g}^{-1}$ de substrat.

\section{Statistiques}

Les dispositifs expérimentaux étaient des plans en blocs aléatoires complets. $L$ 'analyse statistique a été faite avec une procédure générale des modèles linéaires avec SAS (version 6.08) (SAS inc., Cary, NC). Les données en pourcentage ont été traitées avec la transformation arcsin et le poids frais de la partie aérienne avec une transformation racine carrée avant les analyses. Les moyennes ont été séparées selon la méthode LSD de Fisher $(P<0.05)$. 


\section{RÉSULTATS}

Effet de la concentration en spores de Trichoderma harzianum MAUL20 sur le concombre et la tomate La concentration en spores de $T$. harzianum MAUL-20 dans le substrat n'a pas influencé le pourcentage de germination des plants de concombre ( $\mathrm{Ta}$ bleau 1). Par contre, chez la tomate, la plus forte concentration $\left(10^{7} \mathrm{cfu} \mathrm{g}^{-1}\right)$ a amélioré la germination des plantes. Au niveau de la hauteur et du poids frais de la partie aérienne des plantes, les concentrations $10^{5} \mathrm{cfu} \mathrm{g}^{-1}$ et supérieures ont toutes significativement bonifié ces variables et ce, tant chez le concombre que la tomate (Tableau 1).

\section{Activité antagoniste de Trichoder- ma harzianum MAUL-20 contre cinq champignons telluriques phytopa- thogènes}

L'effet des agents pathogènes FORL, $P$. ultimum, $R$. solani, $S$. sclerotiorum et $V$. dahliae sur la germination des graines de concombre et de tomate a été déterminé après $30 \mathrm{j}$ de croissance en serre. Le pourcentage de germination tient compte des cas de fonte des semis en pré et post-émergence. Chez le concombre, seul S. sclerotiorum (Ss-) a réduit de façon significative la germination des plants (Fig. 1 A). En ce qui concerne la hauteur et le poids frais de la partie aérienne, la présence de $T$. harzianum a amélioré les valeurs des deux variables dans les traitements témoin, $\mathrm{Fo}+$, Pu+ et Rs+ (Fig. $1 \mathrm{~B}$ et $\mathrm{C}$ ). Par contre, lorsque comparé au Tem, la présence $d^{\prime}$ agents pathogènes (cas -) a significativement réduit la hauteur, le poids frais de la partie aérienne et augmenté l'index de la gravité de la maladie des plants sauf dans les cas de Fo- (ce champignon n'étant pas pathogène chez le concombre) et dans le cas de Ss- (index de la gravité de la maladie) (Fig. 1 B - D).

Chez la tomate, seuls les traitements avec $P$. ultimum ( $\mathrm{Pu}$-) et $S$. sclerotiorum (Ss-) ont significativement affecté la germination des plants (Fig. 2 A). Concernant la hauteur et le poids frais de la partie aérienne, la présence de $T$. harzianum a amélioré les valeurs des deux paramètres de croissance dans les traitements $\mathrm{Fo}+, \mathrm{Pu}+$ et $\mathrm{Rs}+$ (Fig. $2 \mathrm{~B}$ et $\mathrm{C}$ ). Par contre, l'index de la gravité de la maladie a démontré un effet positif de $T$. harzianum contre tous les agents pathogènes sauf dans le cas de Fo+ (Fig. 2 D). Lorsque comparé au Tem, la présence $d^{\prime}$ agents pathogènes (cas -) a significativement réduit la hauteur, le poids frais de la partie aérienne et augmenté l'index de la gravité de la maladie des plants sauf dans le cas de Fo(Fig. 2 B - D).

Tableau 1. Effet de la concentration en spores de Trichoderma harzianum MAUL-20 sur la croissance de plants de concombre et tomate

\begin{tabular}{|c|c|c|c|c|c|c|}
\hline \multirow{2}{*}{ 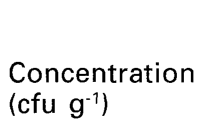 } & \multicolumn{2}{|c|}{$\begin{array}{c}\text { Pourcentage } \\
\text { de germination }\end{array}$} & \multicolumn{2}{|c|}{ Hauteur $(\mathrm{cm})$} & \multicolumn{2}{|c|}{$\begin{array}{l}\text { Poids frais de la } \\
\text { partie aérienne }(\mathrm{g})\end{array}$} \\
\hline & Concombre & Tomate & Concombre & Tomate & Concombre & Tomate \\
\hline $10^{0}$ & $96 a^{a}$ & 87,5 a & 5,38 a & $4,57 \mathrm{a}$ & $13,24 \mathrm{a}$ & 3,39 a \\
\hline $10^{4}$ & $100 \mathrm{a}$ & $91,5 \mathrm{ab}$ & $5,65 a$ & $4,94 \mathrm{a}$ & $14,41 \mathrm{a}$ & $3,94 \mathrm{a}$ \\
\hline $10^{5}$ & $100 \mathrm{a}$ & $95,8 \mathrm{ab}$ & $6,06 \mathrm{~b}$ & $5,45 b$ & $16,13 \mathrm{~b}$ & $4,33 a b$ \\
\hline $10^{6}$ & $100 \mathrm{a}$ & $96,0 a b$ & $6,46 \mathrm{c}$ & $5,80 \mathrm{~b}$ & $16,63 \mathrm{~b}$ & $5,62 \mathrm{~b}$ \\
\hline $10^{7}$ & $100 \mathrm{a}$ & $98,0 \mathrm{~b}$ & $7,05 \mathrm{~d}$ & $6,64 \mathrm{c}$ & $18,39 \mathrm{c}$ & $8,91 \mathrm{c}$ \\
\hline
\end{tabular}

a Pour une même colonne, les valeurs suivies de la même lettre ne sont pas significativement différentes à un seuil de 0.05 , selon le test LSD de Fisher. 

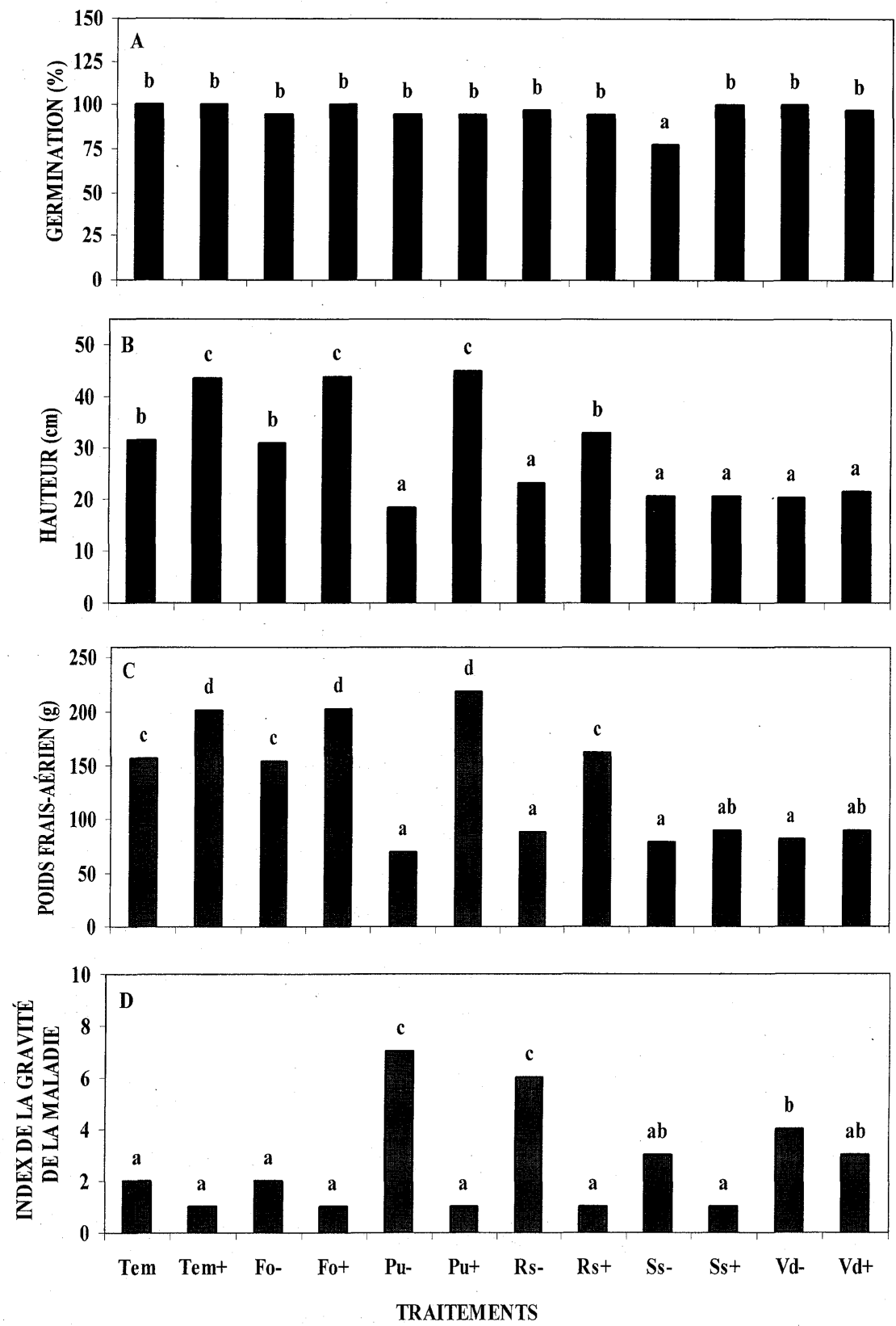

Figure 1. Effet de Trichoderma harzianum MAUL-20 sur (A) le pourcentage de germination, (B) la hauteur, (C) le poids frais de la partie aérienne et (D) l'indice de la gravité de la maladie des plants de concombre lorsque confrontés à divers agents pathogènes. Les valeurs suivies de la même lettre ne sont pas significativement différentes à un seuil de 0.05 , selon le test LSD de Fisher. Tem = Substrat + plante; Tem + = Substrat + Trichoderma harzianum MAUL20 + plante; Fo = Fusarium oxysporum f. sp. radicis-lycopersici; $\mathbf{P u}=$ Pythium ultimum; $\mathbf{R s}=$ Rhizoctonia solani; $\mathbf{S s}=$ Sclerotinia sclerotiorum; Vd = Verticillium dahliae; - = présence de l'agent pathogène dans le substrat; + = présence de l'agent pathogène et de Trichoderma harzianum MAUL-20 dans le substrat. 

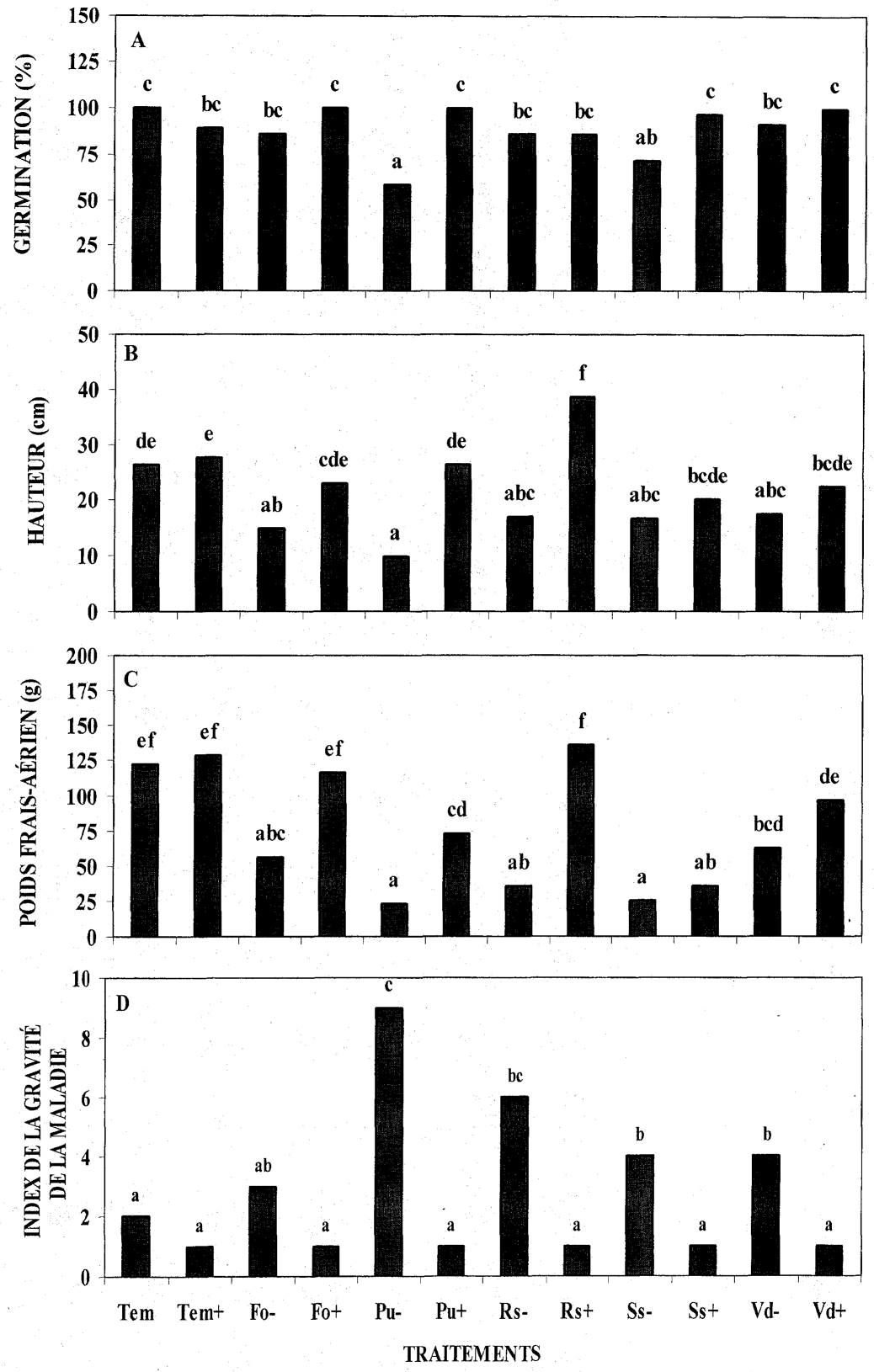

Figure 2. Effet de Trichoderma harzianum MAUL-20 sur (A) le pourcentage de germination, (B) la hauteur, (C) le poids frais de la partie aérienne et (D) I'indice de la gravité de la maladie des plants de tomate lorsque confrontés à divers agents pathogènes. Les valeurs suivies de la même lettre ne sont pas significativement différentes à un seuil de 0.05 , selon le test LSD de Fisher. Tem = Substrat + plante; Tem + = Substrat + Trichoderma harzianum MAUL20 + plante; Fo = Fusarium oxysporum f. sp. radicis-lycopersici; $\mathbf{P u}=$ Pythium ultimum; $\mathbf{R s}=$ Rhizoctonia solani; $\mathbf{S s}=$ Sclerotinia sclerotiorum; Vd = Verticillium dahliae; $-=$ présence de l'agent pathogène dans le substrat; + = présence de l'agent pathogène et de Trichoderma harzianum MAUL-20 dans le substrat. 


\section{Comparaison de l'activité biolo- gique de Trichoderma harzianum MAUL-20 et du biofongicide Rootshield ${ }^{\mathrm{TM}}$}

L'effet des agents pathogènes FORL, $P$. ultimum, $R$. solani, $S$. sclerotiorum et $V$. dahliae, sur la germination des graines de concombre et de tomate, a été évalué après $30 \mathrm{j}$ de croissance en serre. Le pourcentage de germination tient compte des cas de fonte des semis en pré- et post-émergence. Chez le concombre, aucun traitement n'a affecté la germination des plants (Fig. 3 A). En ce qui concerne la hauteur et le poids frais de la partie aérienne des plants, la présence de Trichoderma a amélioré les valeurs des deux variables dans les traitements témoins, $\mathrm{Pu}-20$ et Rs (20 et Ro) tandis que $\mathrm{Pu}$-Ro a significativement augmenté le poids frais de la partie aérienne des plantes seulement (Fig. 3 $B$ et $C)$. Aucune différence significative n'a été observée entre les deux témoins Trichoderma. Lorsque les deux biofongicides sont comparés, seul $\mathrm{Pu}-20$ a donné de meilleurs résultats.

Chez la tomate, seul $R$. solani a réduit de façon significative la germination des plants (Fig. 4 A). En ce qui concerne la hauteur et le poids frais de la partie aérienne des plants, la présence de Trichoderma a amélioré les valeurs des deux paramètres dans les traitements $\mathrm{Pu}-\mathrm{Ro}, \mathrm{Rs}-20$ et Rs-Ro lorsque comparé au Tem tandis que Pu-20 a significativement augmenté le poids frais de la partie aérienne des plantes seulement (Fig. 4 $B$ et $C$ ). Lorsque comparés, aucune différence significative $n^{\prime}$ a été observée entre les deux biofongicides.

\section{DISCUSSION}

Les résultats de cette recherche ont démontré que la souche de $T$. harzianum MAUL-20 peut avoir certains effets bénéfiques sur la croissance des plants de concombre et de tomate lorsqu'ajoutée aux substrats de culture. Des effets similaires de stimulation de croissance ont déjà été rapportés avec le biofongicide Rootshield et d'autres souches de Trichoderma (Baker 1988; Kleifield et Chet 1992; Windham et al. 1986) et pourraient être induits par a) une production d'hormones de la part de la plante (Chang et al. 1986; Windham et al. 1986); b) une conversion d'éléments du sol ou de la matière organique en une forme assimilable par la plante (Barber et Lynch 1977); c) une meilleure absorption et translocation des éléments minéraux (Kleifield et Chet 1992); et d) un contrôle des agents pathogènes mineurs de la rhizosphère dans le cas des sols (Broadbent et al. 1977; Elad et al. 1987).

Les résultats démontrent qu'un agent de lutte biologique ne peut être efficace contre tous les agents pathogènes d'une culture (Dennis et Webster 1971a). Chez le concombre, les résultats indiquent que la fonte des semis provoquée par Pythium et le rhizoctone commun induit par $R$. solani peuvent être contrôlés par T. harzianum MAUL-20 tandis que chez la tomate, le flétrissement fusarien causé par FORL et la fonte des semis causée par $P$. ultimum et $R$. solani peuvent également être contrôlés par $T$. harzianum MAUL-20. Le FORL n'est pas un agent pathogène du concombre et les résultats corroborent ce fait puisque FORL- n'a pas influencé le développement de la plante et l'ajout de $T$. harzianum MAUL-20 au substrat a eu le même effet que le témoin positif (Tem+). L'inaction de cet agent pathogène sur le concombre est venue valider les résultats obtenus avec les deux témoins.

Dans cette étude, T. harzianum MAUL-20 a eu un effet protecteur et stimulateur de croissance dans les cas de $P$. ultimum et $R$. solani chez le concombre et la tomate puisque aucun cas de fonte des semis n'a été constaté dans 

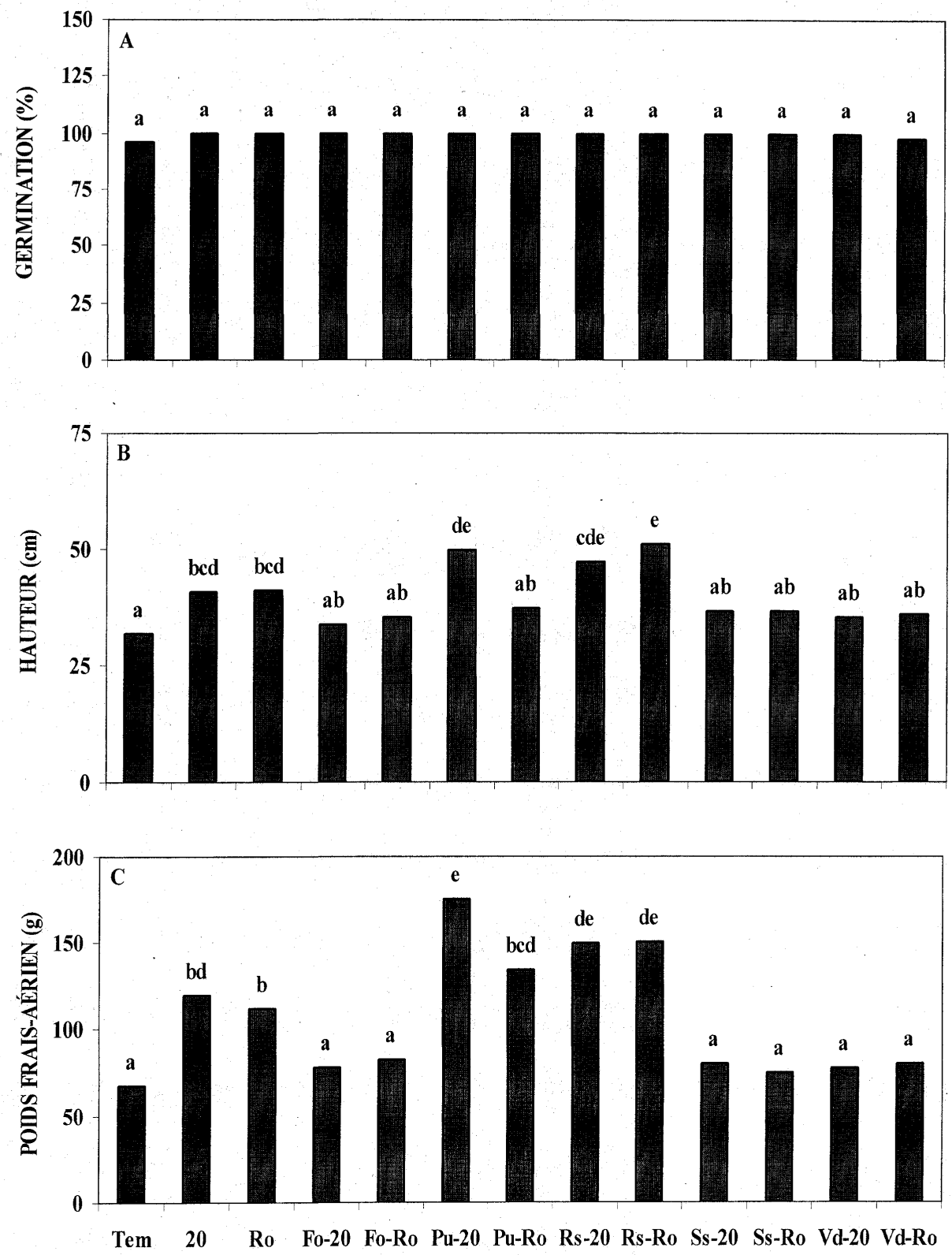

TRAITEMENTS

Figure 3. Effet de la souche de Trichoderma harzianum MAUL-20 et du biofongicide Rootshield sur (A) le pourcentage de germination, (B) la hauteur et (C) le poids frais de la partie aérienne des plants de concombre. Les valeurs suivies de la même lettre ne sont pas significativement différentes à un seuil de 0.05 , selon le test LSD de Fisher. Tem $=$ Substrat + plante; $20=$ Substrat + Trichoderma harzianum MAUL-20 + plante; Ro = Substrat + Rootshield + plante; Fo = Fusarium oxysporum f. sp. radicis-lycopersici; $\mathbf{P u}=$ Pythium ultimum; $\mathbf{R s}=$ Rhizoctonia solani; $\mathbf{S s}=$ Sclerotinia sclerotiorum; $\mathbf{V d}=$ Verticillium dahliae . 

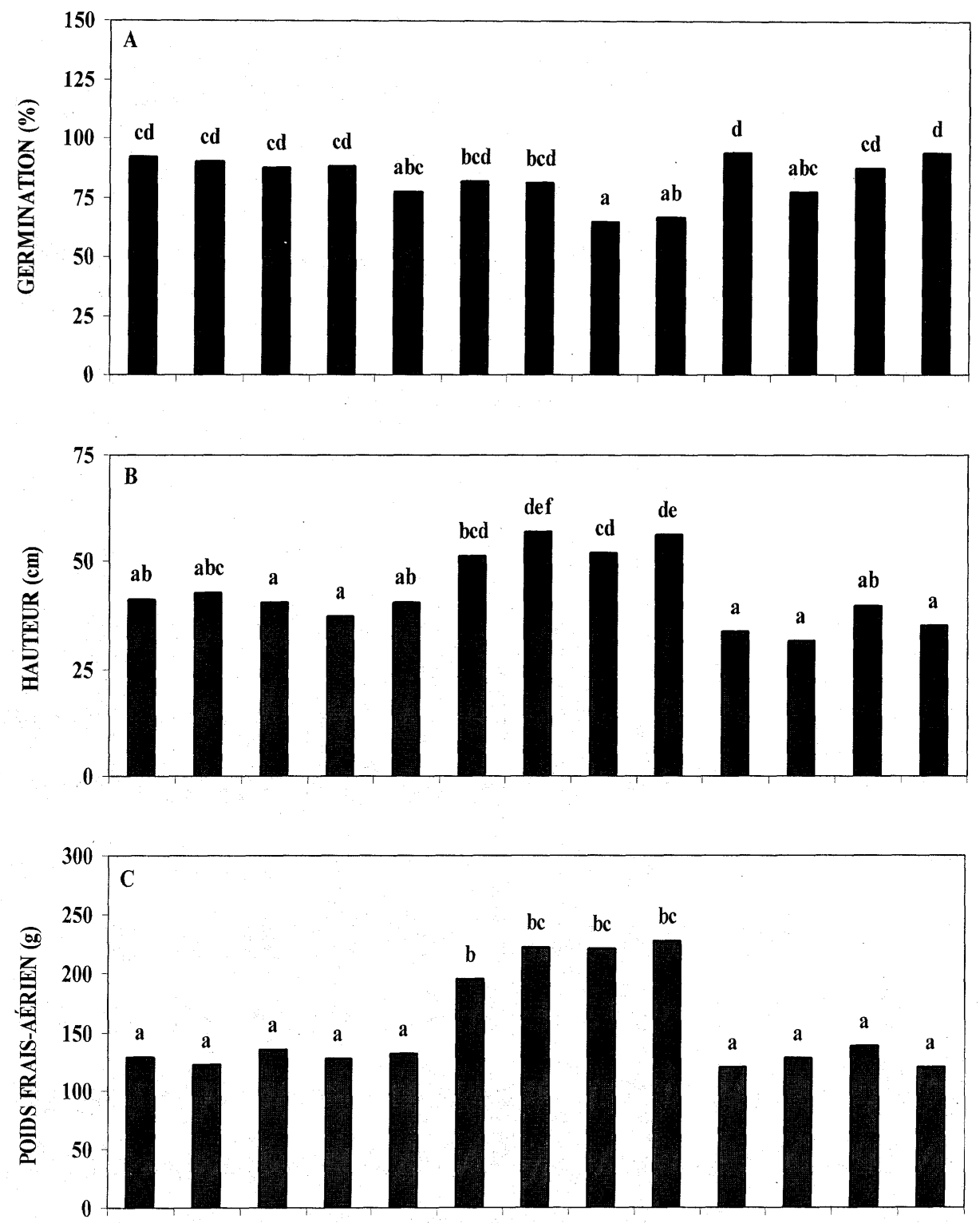

$\begin{array}{llllllll}\text { Tem } & 20 & \text { Ro } & \text { Fo-20 Fo-Ro Pu-20 Pu-Ro Rs-20 Rs-Ro } & \text { Ss-20 Ss-Ro Vd-20 Vd-Ro }\end{array}$

TRAITEMENTS

Figure 4. Effet de la souche de Trichoderma harzianum MAUL-20 et du biofongicide Rootshield sur (A) le pourcentage de germination, (B) la hauteur et (C) le poids frais de la partie aérienne des plants de tomate. Les valeurs suivies de la même lettre ne sont pas significativement différentes à un seuil de 0.05 , selon le test LSD de Fisher. Tem $=$ Substrat + plante; $20=$ Substrat + Trichoderma harzianum MAUL-20 + plante; $\mathbf{R o}=$ Substrat + Rootshield + plante; $\mathbf{F o}=$ Fusarium oxysporum f. sp. radicis-lycopersici; $\mathbf{P u}=$ Pythium ultimum; $\mathbf{R s}=$ Rhizoctonia solani; $\mathbf{S s}=$ Sclerotinia sclerotiorum; $\mathbf{V d}=$ Verticillium dahliae. 
ces deux traitements comparativement aux Pu- et Rs-. Par contre, chez le concombre, I'action de T. harzianum MAUL20 sur $P$. ultimum a été plus importante que sur $R$. solani puisqu'elle a permis $d^{\prime}$ atteindre un niveau similaire au Tem+ dans le cas de $P$. ultimum et seulement la performance du Tem dans le cas de $R$. solani. Cette constatation pourrait indiquer que la lutte contre $R$. solani a été plus difficile que celle contre $P$. ultimum. Chez la tomate, la réaction inverse a été notée. Néanmoins, cette action de $T$. harzianum MAUL-20 sur Pythium, responsable de la fonte des semis sur les jeunes plantules, et sur $R$. solani, agent de la même maladie mais sur des plantules plus âgées, constitue un net avantage pour ces cultures en conditions contrôlées (Bates et Stanghellini 1984; Favrin et al. 1988; Larkin et Fravel 1998). Chez la tomate de serre, les symptômes induits par FORL surviennent principalement lorsque les plants sont matures mais ils peuvent également affecter sévèrement les jeunes plantules (Jarvis 1988). Un effet positif de l'action de Trichoderma a été observé contre FORL, puisque les variables de croissance ont atteint celles du Tem+ Iorsque T. harzianum MAUL-20 était présent. Cette activité antagoniste de $T$. harzianum MAUL-20 contre FORL est très importante considérant les fortes pertes causées par ce champignon sur les cultures de tomate en serre (Jarvis 1988; Menzies et al. 1990). De plus, le contrôle de FORL dans les serres limitera sa dispersion par des vecteurs potentiels comme la mouche du terreau (Bradysia spp. [Diptera : Sciaridae] (Gillepsie et Menzies 1993) et permettra de restreindre l'utilisation des fongicides qui sont peu ou pas efficaces.

Chez le concombre, $T$. harzianum MAUL-20 n'a pas été en mesure de compétitionner avec $S$. sclerotiorum et $V$. dahliae puisque l'effet positif généré par T. harzianum MAUL-20 avec les autres champignons pathogènes $n^{\prime} a$ pas été noté dans ces deux cas. Pourtant, in vitro, $T$. harzianum MAUL-20 avait montré une vitesse de croissance de deux à sept fois supérieure à celle de $S$. sclerotiorum et $V$. dahliae respectivement et une activité antagoniste importante contre $S$. sclerotiorum mais mi- tigée contre $V$. dahliae (données non fournies). La souche de S. sclerotiorum utilisée devait être très agressive dans le substrat puisque la croissance des plantes a été nettement ralentie lorsque comparée au Tem. Paulitz et al. (1990) mentionnent que la constitution d'un substrat peut favoriser le développement de certains agents pathogènes au détriment de l'agent biologique. L'agent pathogène profiterait alors de la compétition pour les éléments nutritifs disponibles dans le substrat pour accroître son potentiel d'inoculum et par conséquent, sa capacité à causer la maladie. Pourtant, la réponse aurait dû être à I'opposé de celle obtenue puisque les symptômes induits par $S$. sclerotiorum apparaissent surtout à la floraison et davantage lorsqu'il y a une mauvaise gestion de l'eau et que la densité des plants est trop élevée, ce qui n'était pas le cas dans ces expériences (Richard et Boivin 1994). De plus, cette souche de $S$. sclerotiorum a été isolée de céréales, ce qui aurait pu nuire à son développement dans les substrats (Cook 1993) et elle a été introduite au substrat sous forme de fragments d'hyphes et de sclérotes, des moyens de contamination moins efficaces que les spores ou conidies (Agrios 1988).

Chez la tomate, l'ajout de T. harzianum MAUL-20 au substrat a permis d'atteindre le niveau des Tem et Tem+ dans les cas de V. dahliae et $S$. sclerotiorum (sauf pour Ss+, poids frais de la partie aérienne) même si les résultats ne sont pas significativement différents de Ss- et Vd-. Tout comme pour le concombre, $T$. harzianum MAUL-20 a eu de la difficulté à contrer ces deux agents pathogènes, qui sont pourtant reconnus pour être très agressifs chez la tomate de champ et à un degré moindre chez la tomate de serre (Abawi et Grogan 1979; Hawksworth et Talboys 1970; Purdy 1979).

L'introduction de l'agent de lutte biologique au substrat au moins 2 semaines avant l'arrivée de l'agent pathogène est I'approche à privilégier (Alabouvette et al. 1993; Lewis et al. 1998). Durant cette période, Trichoderma se multiplie et colonise la rhizosphère ce qui lui permet d'occuper prioritairement la niche 
écologique. II met alors à profit l'un de ses modes d'action, la compétition ( $\mathrm{Pa}$ pavizas 1985). Dans le cas du concombre, les résultats révèlent que $T$. harzianum MAUL-20 a un effet stimulant sur la croissance de la plante $(\mathrm{Tem}+)$. Cet effet stimulant n'a pas été observé chez la tomate. Cette stimulation se traduirait par un développement accru de la plante et il concerne non seulement la partie aérienne des plantes mais aussi le système racinaire (données non incluses), ce qui s'est soldé par un index de la sévérité de la maladie inférieur à 2 .

Les tests effectués avec $T$. harzianum MAUL-20 ont démontré une efficacité similaire et, dans certains cas, supérieure au biofongicide Rootshield. Cette constatation est très importante puisque notre biofongicide T. harzianum MAUL20 a été appliqué à une concentration qui est 10 fois inférieure à celle recommandée pour Rootshield (Harman 2000; Larkin et Fravel 1998). De plus, Rootshield est issu d'une fusion protoplasmique de deux souches de Trichoderma harzianum (Harman 2000; Stasz et al. 1988) tandis que T. harzianum MAUL20 est une souche indigène au Québec. Plusieurs recherches mentionnent qu'une souche indigène de Trichoderma n'est pas aussi bonne compétitrice de la rhizosphère que celles ayant reçu un caractère spécifique (Ahmad et Baker 1988; Papavizas 1985). Nos données démontrent un effet stimulant de T. harzianum MAUL-20 sur le développement de la plante et de son système racinaire, ce qui se traduit par un développement aérien accru et une meilleure compétitivité de la plante lorsque confrontée à différents agents pathogènes ( $P$. ultimum, $R$. solani et FORL). Ces résultats rejoignent ceux énoncés par Sivan and Chet (1989) qui ont démontré qu'une souche indigène de Trichoderma pouvait très bien se développer et compétitionner dans la rhizosphère.

Afin de mieux comprendre le complexe Plante - Agent pathogène - Trichoderma, I'utilisation de substrats commerciaux devrait être privilégiée puisqu'ils sont vendus comme étant exempts d'agents pathogènes et de mauvaises herbes. II serait alors plus facile de déterminer l'action de Trichoderma sur la plante, sur l'agent pathogène seul et sur la dualité existant entre agent pathogène et agent biologique. De plus, certains de ces substrats sont riches en cellulose ce qui favoriserait la multiplication de Trichoderma et se traduirait par une meilleure agressivité et un pouvoir de compétition amélioré dans la rhizosphère, puisqu'il est reconnu comme un excellent dégradeur de ce composé (Papavizas 1985). Tout le marché de la production de transplants pour le champ, le domaine serricole et la production ornementale (fleurs annuelles, plantes vivaces, potées fleuries, etc.) pourrait en profiter. L'utilisation de Trichoderma à des fins de lutte biologique aurait des répercussions positives sur l'environnement: diminution des intrants chimiques, amélioration des rendements, puisque les plantes seraient moins stressées par l'action souvent nocive des fongicides, et réduction de l'exposition des travailleurs à ces produits.

\section{REMERCIEMENTS}

Nous remercions M. François Charrier et $M$. Irénée Couture pour leur support technique lors des essais en serre. Ce projet a été financé par le Fonds des priorités gouvernementales en sciences et en technologie - Volet Environnement (FPGST-E).

\section{RÉFÉRENCES}

Abawi, G.S. et R.G. Grogan. 1979. Epidemiology of diseases caused by Sclerotinia species. Phytopathology 69 : 899-904.

Adams, P.B. 1990. The potential of mycoparasites for biological control of plant diseases. Annu. Rev. Phytopathol. 28 : 5972.

Agrios, G.N. 1988. Plant Pathology. $3^{e}$ éd. Academic Press, New-York. 803 pp.

Ahmad, J.S. et R. Baker. 1988. Implications of rhizosphere competence of Trichoderma harzianum. Can. J. Microbiol. 34 : 229-234.

Alabouvette, C., P. Lemanceau et C. Steinberg. 1993. Recent advances in the biological control of Fusarium wilts. Pestic. Sci. 37 : 365-373. 
Anonyme. 1992. Noms des maladies des plantes au Canada. Société de Protection des Plantes du Québec. 477 pp.

Backman, P.A. et R. Rodriguez-Kabana. 1975. A system for the growth delivery of biological control agents to the soil. Phytopathology $65: 819-821$.

Baker, R. 1988. Trichoderma spp. as plantgrowth stimulants. CRC Crit. Rev. Biotechnol. 7 : 97-106.

Barber, D.A. et J.M. Lynch. 1977. Microbial growth in the rhizosphere. Soil Biol. Biochem. $9: 305-308$.

Bates, M.L. et M.E. Stanghellini. 1984. Root rot of hydroponically grown spinach caused by Pythium aphanidermatum and $P$. dissocotum. Plant Dis. 68 : 989-991.

Bélanger, R.R. et C. Labbé. 1994. Commercial-scale control of rose powdery mildew with a fungal antagonist. Plant Dis. $78: 420-424$.

Besnard, O. et P. Davet. 1993. Mise en évidence de souches de Trichoderma spp. à la fois antagonistes de Pythium ultimum et stimulatrices de la croissance des plantes. Agronomie $13: 413-421$.

Boland, G.J. 1990. Biological control of plant diseases with fungal antagonists : Challenges and opportunities. Can. J. Plant Pathol. 12 : 295-299.

Bolton, A.T. 1980. Control of Pythium aphanidermatum in poinsettia in a soilless culture by Trichoderma viride and Streptomyces spp. Can. J. Plant Pathol. 2 : 9395.

Broadbent, P., K.F. Baker, N. Franks et J. Holland. 1977. Effet of Bacillus on increased growth seedlings in steamed and nontreated soil. Phytopathology 67 : 1027 1033.

Brodeur, J., A. Bouchard et G. Turcotte. 1997. Potential of four species of predator mites as biological control agents of the tomato russet mite, Aculops lycopersici (Massee) (Eriophyidae). Can. Entomol. 129 : $1-6$.

Caron, J. 1993. Isolement et caractérisation de divers isolats de Trichoderma comme agent de lutte biologique contre la moisissure grise (Botrytis cinerea) dans la production de la fraise. Thèse M.Sc. no 12 350, Université Laval, Québec. 121 pp.

Chang, Y.-C., Y.-C. Chang et R. Baker. 1986. Increased growth of plants in the presence of the biological control agent Trichoderma harzianum. Plant Dis. 70 : 145148.

Chet, I. et R. Baker. 1981. Isolation and biocontrol potential of Trichoderma hamatum from soil naturally suppressive to Rhizoctonia solani. Phytopathology 71 : 286-290.
Chet, I., G.E. Harman et R. Baker. 1981. Trichoderma harzianum : Its hyphal interactions with Rhizoctonia solani and Pythium spp. Microbiol. Ecol. 7 : 29-38.

Clavet, C., J. Pera et J.M. Barea. 1993. Growth response of marigold (Tagetes erecta L.) to inoculation with Glomus mosseae, Trichoderma aureoviride and Pythium ultimum in a peat-perlite mixture. Plant Soil $148: 1-6$.

Cook, R.J. 1993. Making greater use of introduced microorganisms for biological control of plant pathogens. Annu. Rev. Phytopathol. 31 : 53-80.

Dennis, L. et J. Webster. 1971a. Antagonistic properties of species-groups of Trichoderma. II. Production of volatile antibiotics. Trans. Br. Mycol. Soc. 57 : 41-48.

Dennis, L. et J. Webster. 1971b. Antagonistic properties of species-groups of Trichoderma. III. Hyphal interaction. Trans. Br. Mycol. Soc. 57 : 363-369.

De Waard, M.A., S.G. Georgopoulos, D.W. Hollomon, H. Ishii, P. Leroux, N.N. Ragsdale et F.J. Schwinn. 1993. Chemical control of plant diseases: problems and prospects. Annu. Rev. Phytopathol. 31 : 403-421.

Elad, Y., I. Chet et J. Katan. 1980. Trichoderma harzianum : A biocontrol agent effective against Sclerotinia rolfsii and Rhizoctonia solani. Phytopathology 70 : 119121.

Elad, Y., I. Chet et Y. Henis. 1982. Degradation of plant pathogenic fungi by Trichoderma harzianum. Can. J. Microbiol. 28 : 719-725.

Elad, Y., I. Chet et R. Baker. 1987. Increased growth response of plants induced by rhizobacteria antagonistic to soilborne pathogenic fungi. Plant Soil $98: 325-330$.

Favrin, R.J., J.E. Rahe et B. Mauza. 1988. Pythium spp. associated with crown rot of cucumbers in British Columbia greenhouse. Plant Dis. 72 : 683-687.

Gill, S. et J. Sanderson. 1998. Ball identification guide to greenhouse pests and beneficials. Ball Publishing, Illinois. 244 pp.

Gillepsie, D.R. et J.G. Menzies. 1993. Fungus gnats vector Fusarium oxysporum f. sp. radicis-lycopersici. Annu. Appl. Biol. 123 : 539-544.

Harman, G.E. 1992. Development and benefits of rhizosphere competent fungi for biological control of plant pathogens. J. Plant Nutr. $15: 835-843$.

Harman, G.E. 2000. Myths and dogmas of biocontrol - changes in perceptions derived from research on Trichoderma harzianum T-22. Plant Dis. 84 : 377-393. 
Hawksworth, D.L. et P.W. Talboys. 1970. Verticillium dahliae. CMI descriptions of pathogenic fungi and bacteria, No 256. Commonw. Mycol. Inst., Kew, Surrey, England, 2 pp.

Jarvis, W.R. 1988. Fusarium crown and root rot of tomatoes. Phytoprotection $69: 49-64$.

Jarvis, W.R. 1993. Managing diseases in greenhouse crops. $2^{\mathrm{e}}$ éd. APS Press, Minnesota. 288 pp.

Jeffers, S.N. et S.B. Martin. 1986. Comparaison of two media selective for Phytophthora and Pythium species. Plant Dis. 70 : 1038-1043.

Kelley, W.D. 1976. Evaluation of Trichoderma harzianum - impregnated clay granules as a biocontrol for Phytophthora cinnamoni causing damping-off of pine seedlings. Phytopathology $66: 1023-1027$.

Kleifeld, O. et I. Chet. 1992. Trichoderma harzianum interaction with plants and effect on growth response. Plant Soil 144 : 267-272.

Larkin, R.P. et D.R. Fravel. 1998. Efficacy of various fungal and bacterial biocontrol organisms for control of Fusarium wilt of tomato. Plant Dis. 82 : 1022-1028.

Lewis, J.A. et G.C. Papavizas. 1987. Application of Trichoderma and Gliocladium in alginate pellets for controls of Rhizoctonia solani damping-off. Plant Pathol. 36 : 438-446.

Lewis, J.A., R.P. Larkin et D.L. Rogers. 1998. A formulation of Trichoderma and Gliocladium to reduce damping-off caused by Rhizoctonia solani and saprophytic growth of the pathogen in soilless Mix. Plant Dis. 82 : 501-506.

Lockwood, J.L. 1988. Evolution of concept associated with soilborne plant pathogens. Annu. Rev. Phytopathol. 26 : 93121.

Lumsden, R.D. et J.A. Lewis. 1989. Selection, production, formulation and commercial use of plant disease, biocontrol fungi : problems and progress. Pages 171190 in J.M. Whipps et R.D. Lumsden (eds.), Biotechnology of fungi for improving plant growth. Cambridge University Press, Cambridge.

Menzies, J.G., C. Koch et F. Seywerd. 1990. Additions to the host range of Fusarium oxysporum f.sp. radicis-lycopersici. Plant Dis. 74 : 569-572.

Ole Becker, J. et F.J. Schwinn. 1993. Control of soil-borne pathogens with living bacteria and fungi : status and outlook. Pestic. Sci. $37: 355-363$.

Papavizas, G.C. 1985. Trichoderma and Gliocladium: Biology, ecology, and potential for biocontrol. Annu. Rev. Phytopathol. $23: 23-54$
Paulitz, T.C., J.S. Ahmad et R. Baker. 1990. Integration of Pythium nunn and Trichoderma harzianum isolate T-95 for the biological control of Pythium dampingoff cucumber. Plant Soil 121 : 243-250.

Purdy, L.H. 1979. Sclerotinia sclerotiorum : history, diseases and symptomatology, host range, geographic distribution, and impact. Phytopathology $69: 875-880$.

Richard, C. et G. Boivin (éds.). 1994. Concombre, laitue, poivron et tomate de serre. Pages 335-394 in Maladies et ravageurs des cultures légumières au Canada. Société canadienne de phytopathologie et Société d'entomologie du Canada, $\mathrm{Ca}$ nada.

Sippell, D.W., J.G.N. Davidson et R.S. Sadasivaiah. 1985. Rhizoctonia root rot of raspberry in the peace region of Alberta. Can. J. Plant Pathol. 7 : 184-186.

Sivan, A. et I. Chet. 1989. The possible role of competition between Trichoderma harzianum and Fusarium oxysporum on rhizosphere colonization. Phytopathology $79: 198-203$.

Stasz, T.E., G.E. Harman et N.F. Weeden. 1988. Protoplast preparation and fusion in two biocontrol strains of Trichoderma harzianum. Mycologia 80 : 141-150.

Widden, P. et V. Scattolin. 1988. Competitive interactions and ecological strategies of Trichoderma species colonizing spruce litter. Mycologia 80 : 795-803.

Windham, M.T., Y. Elad et R. Baker. 1986. A mechanism for increased plant growth induced by Trichoderma spp. Phytopathology $76: 518-521$. 